

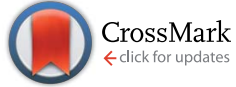

Cite this: Soft Matter, 2014, 10, 6926

\title{
Bending elastic properties of a block copolymer- rich lamellar phase doped by a surfactant: a neutron spin-echo study $\dagger$
}

\author{
H. Egger, $\dot{t}^{a}$ G. H. Findenegg, ${ }^{\star a}$ O. Holderer, ${ }^{b}$ R. Biehl, ${ }^{c}$ M. Monkenbusch ${ }^{c}$ \\ and T. Hellweg ${ }^{\star d}$
}

The influence of the short alkyl-chain ionic surfactant OTAB on the dynamic behavior of an inverse block copolymer-rich lamellar phase was investigated by neutron spin-echo spectroscopy (NSE). The observed intermediate scattering function can be described by a sum of two contributions. For high scattering vectors the model of Zilman-Granek plus a slow diffusional mode can be used to describe the experimental data and the bending elastic modulus $\kappa$ for a polymer-rich membrane is calculated. At low scattering vectors the relaxation curves are strongly influenced by de Gennes narrowing arising from the structure factor of the $L_{\alpha}$ phase. Hence, the computed relaxation rates in this $q$-range are inversely proportional to the static structure factor. The present study demonstrates the necessity to directly investigate the dynamic behavior of lamellar phases and that an analysis of the width of the Bragg peaks can be insufficient to derive information about the single membrane elasticity, especially when both $\kappa$ and $\bar{B}$ depend on the composition of the membrane.

Received 4th March 2014

Accepted 8th May 2014

DOI: $10.1039 / c 4 s m 00494 a$

www.rsc.org/softmatter hydrophobically modified polymers like PAA or PEG;,11-14 amphiphilic poly(ethylene propylene)-poly(ethylene oxide) added to bicontinuous microemulsions; $;^{10,15,16}$ and a triblock-peptide added to a lamellar phase. ${ }^{17}$ An important conclusion of these studies has been that the bending elastic modulus $\kappa,{ }^{18}$ which is of the order of $k_{\mathrm{B}} T$ in such systems, increases with the amount of added block copolymer. For lamellar phases mainly consisting of amphiphilic block copolymers, on the other hand, no NSE measurements have been performed so far and only results obtained by other techniques can be found in the literature. One example is a triblock copolymer from the Pluronics group ${ }^{19}$ which was mixed with small amounts of lipids to produce giant vesicles. These systems were studied using optical microscopy. ${ }^{20}$

In the present work we investigate the effect of a short alkylchain cationic surfactant on the bending elasticity of a block copolymer based lamellar phase in the quaternary system with oil, water and surfactant. Here, the short alkyl-chain cationic surfactant is expected to act in a similar way on the polymer membrane as co-surfactants do in a microemulsion. ${ }^{19}$

\section{Theoretical background}

The width of the Bragg peaks of lamellar phases can be related to the bilayer elasticity ${ }^{21-23}$ in terms of the Caillé-constant

$$
\eta=\frac{q_{0}{ }^{2} k_{\mathrm{B}} T}{8 \pi \sqrt{\kappa \bar{B} / d}},
$$

where $q_{0}$ represents the position of the Bragg peak, $\bar{B}$ the compressibility modulus of the layer stack (layer 
compressibility modulus at constant chemical potential; for details see ref. 24 page 313), and $d$ the layer spacing in the stack. Since $\eta$ depends on the product of $\kappa$ and $\bar{B}$, these two parameters can not be obtained separately from $\eta$. For the present system it was found that the line shape of the Bragg peaks remains unchanged along some trajectories in composition space or changes in an unexpected way, but independent evidence suggested that $\kappa$ and $\bar{B}$ are changing in an anti-parallel manner. ${ }^{25}$ Detailed knowledge about $\kappa$ can be gained by a direct investigation of the bilayer dynamics in an oriented sample using NSE. In the high- $q$ region beyond the second-order Bragg peak, i.e., $q=1.4$ to $2.6 \mathrm{~nm}^{-1}$, single membrane dynamics is responsible for the decay of the intermediate scattering functions (ISFs) as measured with NSE with eventual admixture of Zimm dynamics of polymer segments. Hence, the ZilmanGranek (ZG) model which assumes membrane Zimm dynamics on an ensemble of "free" membrane patches ${ }^{\mathbf{2 6}}$ is expected to be applicable. The curves should then be describable by a stretched exponential function of the type

$$
S(q, t) / S(q)=A \exp \left(-\left(\Gamma_{q_{z}, \mathrm{ZG}} t\right)^{2 / 3}\right)
$$

Within the approximations used in ref. 26 to arrive at a closed analytical expression, the slope $m$ of a plot of $\Gamma_{q_{z}, \mathrm{ZG}}$ vs. $q^{3}$ is connected to the elastic constant $\kappa$ by: $m=0.025 \gamma_{\kappa}\left(\frac{k_{\mathrm{B}} T}{\kappa}\right)^{1 / 2} \frac{k_{\mathrm{B}} T}{\eta_{\mathrm{o}}}$ with $\gamma_{\kappa} \approx 1$ for large $\kappa$, and $\eta_{\mathrm{o}}$ the effective viscosity of the fluid between the membranes. This approach has already been successfully applied to surfactant rich lamellar systems containing low amounts of block copolymers, ${ }^{7-9}$ to bicontinuous microemulsions, ${ }^{27}$ and to vesicles. ${ }^{28,29}$ Due to the high amount of polymer we expect the rigidity to be larger compared to surfactant based systems and therefore, the approximation $\gamma_{\kappa} \approx 1$ should hold in the present case.

\section{Materials and methods}

\subsection{Materials}

The four-component system made up of the triblock-copolymer $\mathrm{EO}_{37}-\mathrm{PO}_{58}-\mathrm{EO}_{37}$, the surfactant $n$-octyltrimethylammonium bromide (OTAB), the oil $o$-xylene, and water, was chosen for this study. At room-temperature and at an oil-to-water mass ratio of $13: 1$, this system shows a polymer-rich extended lamellar phase $\left(\mathrm{L}_{\alpha}\right)$ containing 20 to $45 \mathrm{wt} \%$ polymer and 7 to $13 \mathrm{wt} \%$ surfactant. This phase had been studied by small-angle X-ray scattering ${ }^{25}$ and first-order Bragg peaks of the lamellar phase were observed in a $q$-range $\left(q=\frac{4 \pi}{\lambda} \sin \left(\frac{\Theta}{2}\right)\right)$ between 0.5 to $0.55 \mathrm{~nm}^{-1}$, corresponding to interlamellar spacings, $d$, between 11 and $13 \mathrm{~nm}$. For more details of the phase behaviour see ref. 25 .

\subsection{Methods}

The NSE experiments were performed employing the FRJ2-NSE instrument at the FZ Jülich, using neutrons of wavelength $\lambda=$ $0.8 \mathrm{~nm}$, with $\Delta \lambda=10 \%$ (full width at half maximum). An overall $q$-range $0.27 \leq q \leq 2.6 \mathrm{~nm}^{-1}$ was covered. In the sample cell, the lamellar phase was oriented by means of an array of quartz sheets with $0.25 \mathrm{~mm}$ spacing. The orientation was such that the scattering vector $\vec{q}$ was perpendicular to the lamellar planes, i.e. $\vec{q}=q_{z}$. All measurements were performed with fully deuterated oil $\left(o\right.$-xylene- $\left.\mathrm{d}_{10}\right)$ and otherwise hydrogenated compounds.

\section{Results and discussion}

Four samples of different compositions were studied (see Table 1). The samples a, b, and c have the same polymer-to-(oil + water)-ratio but an increasing surfactant content. The samples b and $\mathrm{d}$ have the same surfactant-to-(oil + water)-ratio but different polymer contents. In all four samples the oil-to-water mass ratio was $13: 1$.

A typical small-angle neutron scattering intensity profile (Fig. 1) shows two pronounced Bragg peaks at $q_{0,1} \approx 0.5 \mathrm{~nm}^{-1}$ and $q_{0,2} \approx 1.0 \mathrm{~nm}^{-1}$, corresponding to an interlamellar spacing $d$ of $c a .12 .5 \mathrm{~nm}$. Fig. 2 shows the normalized ISFs $S(q, t) / S(q)$ for all four samples. The six single curves correspond to the scattering vectors $\left(q=0.5 ; 0.8 ; 1.0 ; 1.4 ; 2.0\right.$ and $\left.2.4 \mathrm{~nm}^{-1}\right)$. For all samples the ISFs at high $q$ decay strongly to values below $15 \%$ within the accessible time window. At high scattering vectors one expects to see single membrane dynamics, whereas at lower scattering vectors the collective dynamics of the interacting membrane stack is important. Therefore, in the discussion below, we consider these two $q$-regions separately.

Using only the ZG model, no satisfactory fit of the data is reached, even employing the exponent as an adjustable parameter. However, the relaxation curves can be fitted by the following model: ${ }^{30}$

Table 1 Composition (in terms of mass fractions $w$ ) of the samples (a) $-(d)$, and values of the bending elasticity modulus $\kappa$ determined by a stretched exponential function according to the ZG theory

\begin{tabular}{|c|c|c|c|c|c|}
\hline Sample & $w_{\mathrm{P}}$ & $w_{\mathrm{S}}$ & $\frac{w_{\mathrm{P}}}{\left(w_{\mathrm{O}}+w_{\mathrm{W}}\right)}$ & $\frac{w_{\mathrm{S}}}{\left(w_{\mathrm{O}}+w_{\mathrm{W}}\right)}$ & $\kappa / k_{\mathrm{B}} T$ \\
\hline (a) & 0.363 & 0.095 & 0.67 & & 8.3 \\
\hline (b) & 0.360 & 0.10 & 0.67 & 0.185 & 5.7 \\
\hline (c) & 0.349 & 0.128 & 0.67 & & 2.7 \\
\hline (d) & 0.265 & 0.115 & & 0.185 & 5.6 \\
\hline
\end{tabular}

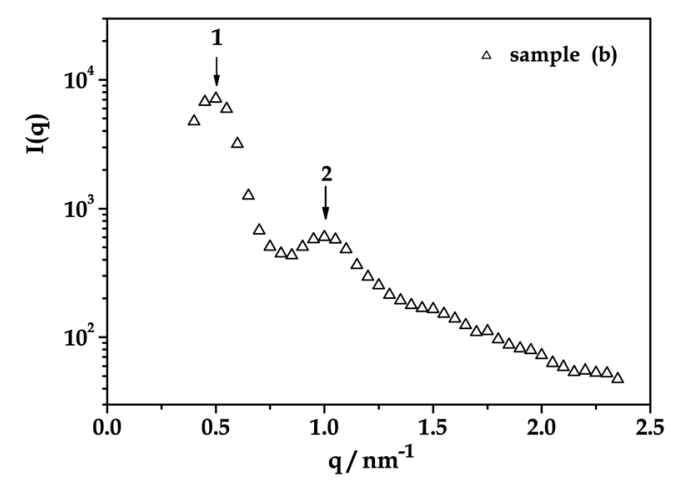

Fig. 1 The static structure factor of the lamellar phase (sample (b)) exhibits two Bragg peaks (first- (1) and second-order (2)). 

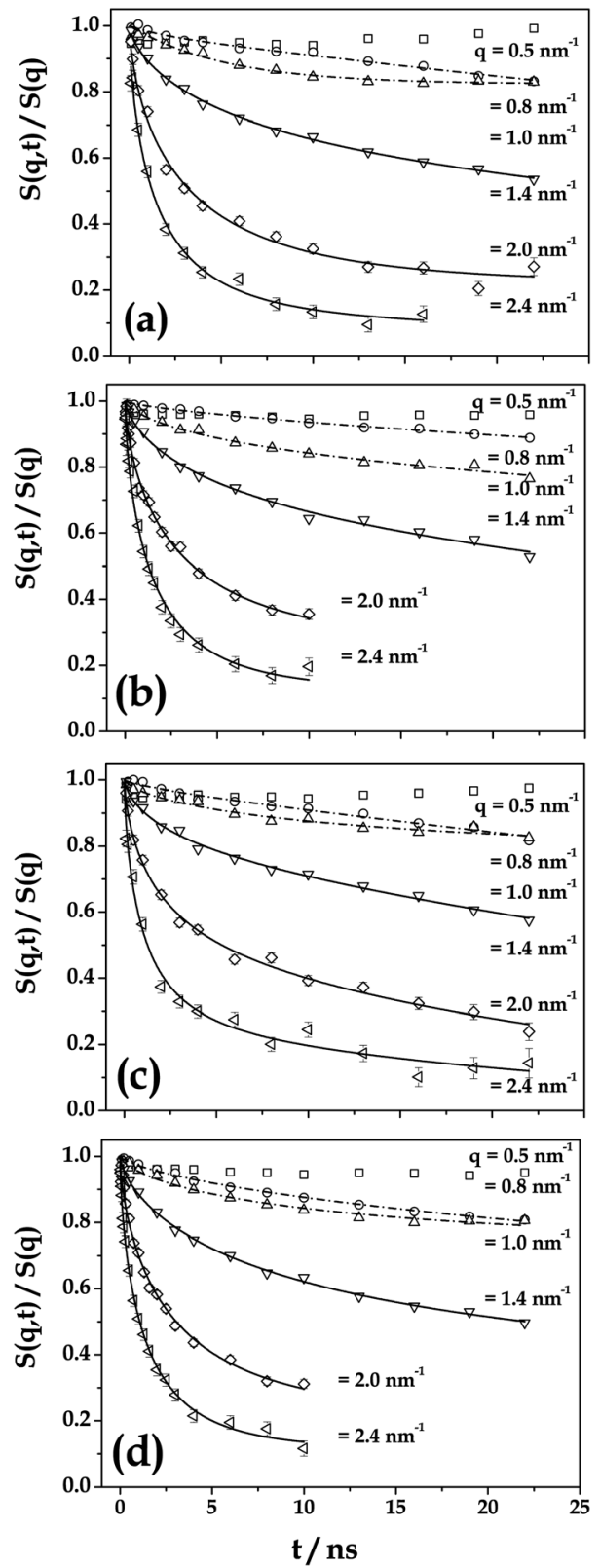

Fig. 2 Normalized NSE intermediate scattering functions $S(q, t) / S(q)$ for the four samples (a)-(d) at six different scattering vectors in the range from $q=0.5$ to $2.4 \mathrm{~nm}^{-1}$. Data for the high- $q$ and low- $q$ regions are analysed separately. The solid lines are best fits for the high- $q$ region, the dash-dotted lines for the low- $q$ region. At $q=1.4 \mathrm{~nm}^{-1}$ the two fits coincide. For details see text

$$
S(q, t) / S(q)=A \exp \left(-\left(\Gamma_{q_{z}, \mathrm{ZG}} t\right)^{\beta_{1}}\right)+(1-A) \exp \left(-\left(\Gamma_{q_{z}, 2} t\right)^{\beta_{2}}\right) .
$$

The parameter $\beta_{1}$ was fixed at $2 / 3$, as the first relaxation process is expected to follow the ZG theory, and $\beta_{2}=1$ was adopted since values near or slightly below 1 were commonly found in an extended analysis. Thus both exponents were fixed and three parameters, the amplitude $A$, and the two relaxation rates $\Gamma_{q_{z}, \mathrm{ZG}}$ and $\Gamma_{q_{z}, 2}\left(\Gamma_{q_{z}, 2}<\Gamma_{q_{z}, \mathrm{ZG}}\right)$ were fitted. The best fits in the high $q$-region are plotted in Fig. 2 as solid lines. The ZG model implies that $\Gamma_{q_{z} \mathrm{ZG}} \sim q^{3}$. Fig. 3 shows that actually all four samples exhibit a slope close to 3 for the relaxation rate $\Gamma_{q_{v}, 1}$ in a $\log -\log$ plot (high frequency). To compute $\kappa$, we assume $\eta_{\mathrm{o}}=$ $0.79 \mathrm{cP}$, i.e. the viscosity of pure $o$-xylene. Additional friction terms due to the polymer decoration of the membrane are neglected. The resulting values of $\kappa$ (Table 1 ) are in a range from ca. 3 to $8 k_{\mathrm{B}}$ T, i.e. higher than those found for polymer-doped surfactant systems $\left(\begin{array}{ll}1-2 & k_{\mathrm{B}} T\end{array}\right)$. This justifies the use of the approximation $\gamma \approx 1$. It has already been shown that the simple relationship between the relaxation rate and $\kappa$, used here, might overestimate $\kappa$, because the assumption $\gamma_{\kappa}=1$ is invalid ${ }^{10}$ if $\kappa$ is low. Complete evaluation of the whole integral expression for lamellar phases ${ }^{31}$ generally yields a more exact value of $\kappa$. However, this requires the additional knowledge or specification of a correlation length $\xi$ which represents the patch size within the Zilman-Granek model. Their approximate analytical expression ignores the influence of $\xi$. It is possible to relate the reduced relaxation rate $\frac{\Gamma_{q_{z}, 1}}{q^{3}} \frac{\eta_{\mathrm{o}}}{k_{\mathrm{B}} T}$, eliminating the viscosity and scattering angle, to the values of $\kappa$ from the whole integral expression, supposed $\xi$ is known.

If the membrane repulsion is governed by the Helfrich steric interaction, $\xi$ is closely related to and of similar magnitude to the layer spacing $d$. Here, $\xi$ is not exactly known, but is expected to be of the order of the interlamellar spacing $d$. This would lead to values of $\kappa$ of the order of $0.5 k_{\mathrm{B}} T$. Note however, that the exact value of $\kappa$ strongly depends on the choice of $\xi$, and is nearly doubled if $\xi$ is increased by a factor of $2(\xi \simeq 2 d)$. However, trends in $\kappa$ resulting from changes in the composition are not affected by this problem and can be discussed independently. Hence, here we will only discuss the outcome obtained using the ZG model.

From a comparison of samples a, b, and $\mathrm{c}$ it is seen that $\kappa$ is decreasing strongly with increasing surfactant concentration. This is consistent with the expectation that a surfactant-rich membrane will be less rigid than a polymer-rich membrane. For the polymer variation (samples b and d) the trend is less pronounced, but it appears that $\kappa$ increases weakly with increasing polymer concentration. Already in our previous work the addition of a surfactant was found to have a strong influence on the shape of the Bragg peaks. However, the observed decrease of the peak width with increasing concentration of OTAB (see Fig. 8 in ref. 25) hinted at surfactant-induced stiffening of the bilayers. This was a counter-intuitive scenario.

The present NSE study indicates the opposite effect and shows that the subsequent addition of OTAB leads to the expected softening of the bilayers. An explanation for this apparent contradiction arises from the fact that the added surfactant is introducing charges into the system. This most likely leads to a change of $\bar{B}$. Since the Bragg peak width depends on both $\kappa$ and $\bar{B}$ in the present case the Caillé approach is not appropriate to draw any conclusions concerning $\kappa$. This is a phenomenon, which was largely ignored in the available literature on bending elastic properties of mixed polymer/ surfactant interfacial films.

The second relaxation rate, $\Gamma_{q_{2}, 2}$, needed to describe the data over the full Fourier time range is roughly one order of magnitude smaller than $\Gamma_{q_{2}, 1}$ and thus the values of $\Gamma_{q_{2}, 2}$ have 


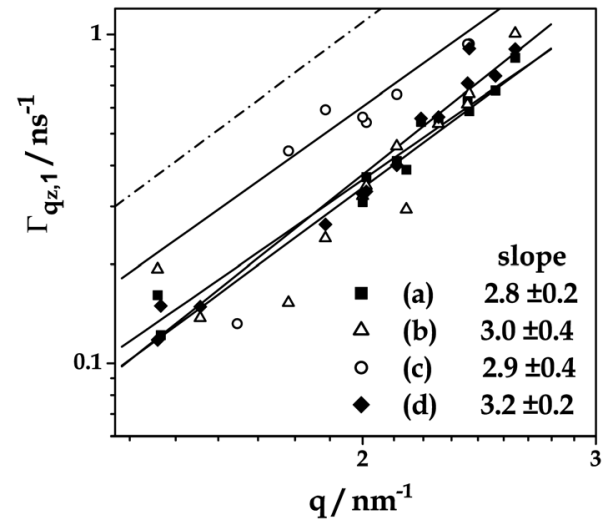

Fig. 3 Double logarithmic plot of the relaxation rate $\Gamma_{q_{z, 1}} v s$. q. All samples exhibit the expected slope of three. For comparison, a dashdotted line with slope three is also shown.

relatively large error limits, which makes it difficult to determine the $q$-dependence of this quantity. A double logarithmic plot gives slopes between two and three (data not shown). This second relaxation contribution can be attributed to the collective breathing modes of the bilayer stack. A linear regression gives a slope corresponding to a collective membrane concentration diffusion coefficient of $D=8( \pm 2) 10^{-12} \mathrm{~m}^{2} \mathrm{~s}^{-1}$.

In the low- $q$ region the static structure factor exhibits two Bragg peaks. The relaxation curves at $q=0.5$ and $1.0 \mathrm{~nm}^{-1}$ correspond to the maxima of the first and second peak. The scattering curves at these two $q$ values show a drastically slower decay, known as de Gennes narrowing ${ }^{32}$ and a quantitative description of the ISFs is difficult to achieve. The ISFs were fitted by the sum of two exponentials:

$$
\begin{aligned}
S(q, t) / S(q)= & {\left[(1-A)+A \exp \left(-\left(\Gamma_{q_{z}, 1}-\Gamma_{q_{z}, 2}\right) t\right)\right] } \\
\times & \exp \left(-\Gamma_{q_{z}, 2} t\right) .
\end{aligned}
$$

The resulting fits are plotted in Fig. 2 as dash-dotted lines. For $q=1.4 \mathrm{~nm}^{-1}$ both fitting functions yield equally good descriptions of the data. All resulting relaxation rates are plotted in Fig. 4(A) together with the respective values for the high $q$-region. Fig. 4(A) indicates that the fast relaxation rate $\Gamma_{q_{z}, 1}$ is a monotonic increasing function of $q$ in the entire experimental $q$ range. The apparent step at $q=1.4 \mathrm{~nm}^{-1}$ is an artifact resulting from the switching of the fit function, i.e. from $\beta_{1}=1$ (low- $q$ region) to $\beta_{1}=2 / 3$ (high- $q$ region). For the slow relaxation rate $\Gamma_{q_{z}, 2}$, the de Gennes narrowing is clearly visible. The dotted lines indicate the position of the Bragg peaks. To indicate more clearly the anomalous behaviour of $\Gamma_{q_{z}, 2}$ in the proximity of the two Bragg peaks a dashed line is drawn as a guide to the eye. Fig. 4(B) shows that a replica of this line perfectly coincides with the static structure factor. However, the de Gennes narrowing makes a clear determination of the general underlying low $q$-dependence impossible.

The amplitude $A$ of eqn (3) is found to increase nearly linearly over the whole $q$-range for all samples (data not shown). This implies that the faster relaxation process becomes more important as $q$ increases. At the highest scattering vectors where
A

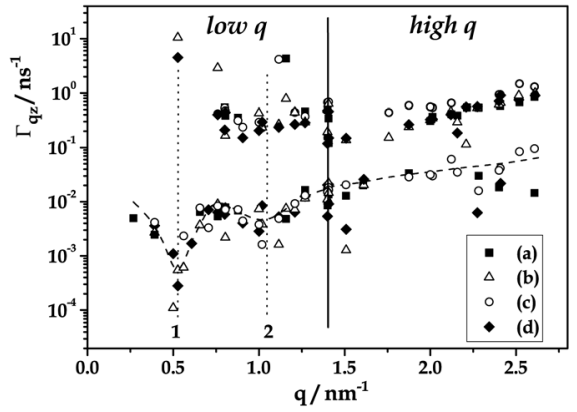

B

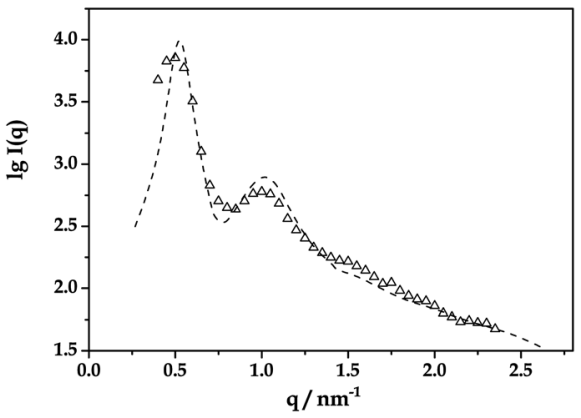

Fig. 4 (A) All relaxation rates as function of $q$. The solid line indicates the border between the low- $q$ and the high- $q$ region, the dotted lines indicate the position of the Bragg peaks. In the slower relaxation process, the de Gennes narrowing is indicated by the dashed line. (B) The reflected image of the dashed line at the abscissa perfectly coincides with the static structure factor.

$A$ is almost 1 the single membrane undulation dominates the relaxation behavior.

\section{Conclusions}

In summary, this study shows that at high scattering vectors, where single membrane dynamics is dominating, the bending elastic modulus $\kappa$ can be determined using the theoretical approach by Zilman and Granek. The resulting values of $\kappa$ show the expected trend with increasing amount of OTAB. The polymer rich bilayer becomes more flexible upon addition of the surfactant. Increasing the polymer mass fraction in the sample yields only a weak increase of $\kappa$. The present work shows that dynamic measurements are necessary to gain complete understanding of the elastic properties of lamellar phases. Static measurements were only suggesting the anti-proportional behavior of $\kappa$ and $\bar{B}$ since the width of the Bragg peaks was found to remain unchanged upon addition of $\mathrm{OTAB}^{25}$ (unchanged or even growing Caillé constant). Knowing the behavior of $\kappa$ with increasing surfactant concentration clearly proves that $\bar{B}$ is increasing with decreasing $\kappa$. Hence, the determination of the Caille constant can be insufficient to derive conclusions about the bilayer elasticity. In the low- $q$ region, the data evaluation is strongly affected by de Gennes narrowing arising from the Bragg peaks of the ordered lamellar phase, and the resulting relaxation rates reflect the static structure factor of the samples. 


\section{References}

1 T. Hellweg and D. Langevin, Phys. Rev. E: Stat., Nonlinear, Soft Matter Phys., 1998, 57, 6825-6834.

2 T. Hellweg, M. Gradzielski, B. Farago and D. Langevin, Colloids Surf., A, 2001, 183-185, 159-169.

3 M. Maugey and A. M. Bellocq, Langmuir, 2001, 17, 67406742.

4 B.-S. Yang, J. Lal, M. Mihailescu, M. Monkenbusch, D. Richter, J. S. Huang, J. Kohn, W. B. Russel and R. K. Prud'homme, Langmuir, 2002, 18, 6-13.

5 C. Frank, R. Strey, C. Schmidt and C. Stubenrauch, J. Colloid Interface Sci., 2007, 312, 76-86.

6 S. Hoehn, C. Schulreich and T. Hellweg, Tenside, Surfactants, Deterg., 2014, 51, 32-39.

7 O. Holderer, H. Freilinghaus, D. Byelov, M. Monkenbusch, J. Allgaier and D. Richter, Z. Phys. Chem., 2010, 224, 243-251.

8 M. Nagao, S. Chawag and T. Hara, Soft Matter, 2011, 7, 65986605.

9 M. C. Watson, Y. Peng, Y. Zheng and F. L. H. Brown, J. Chem. Phys., 2011, 135, 194701.

10 M. Mihailescu, M. Monkenbusch, H. Endo, J. Allgaier, G. Gompper, D. Richter, B. Jakobs, T. Sottmann and B. Farago, J. Chem. Phys., 2001, 115, 9563-9577.

11 Y. Yang, R. Prud'homme, K. M. McGrath, P. Richetti and C. M. Marques, Phys. Rev. Lett., 1998, 80, 2729.

12 B.-S. Yang, J. Lal, P. Richetti, C. M. Marques, W. B. Russel and R. K. Prud'homme, Langmuir, 2001, 17, 5834-5841.

13 B.-S. Yang, J. Lal, J. Kohn, J. S. Huang, W. B. Russel and R. K. Prud'homme, Langmuir, 2001, 17, 6692-6698.

14 X. Hu, R. Biehl, R. K. Prud'homme, M. Monkenbusch and J. Lal, Phys. B, 2004, 350, 217-219.

15 B. Jakobs, T. Sottmann, R. Strey, J. Allgaier, L. Willner and D. Richter, Langmuir, 1999, 15, 6707-6711.

16 H. Endo, M. Mihailescu, M. Monkenbusch, J. Allgaier, G. Gompper, D. Richter, B. Jakobs, R. Strey and I. Grillo, J. Chem. Phys., 2001, 115, 580-600.
17 N. Tsapis, R. Ober, A. Chaffotte, D. E. Warschawski, J. Everett, J. Kauffman, P. Kahn, M. Waks and W. Urbach, Langmuir, 2002, 18, 4384-4392.

18 W. Helfrich, Z. Naturforsch., 1973, 28c, 693-703.

19 T. Hellweg, in Self Organized Nanostructures of Amphiphilic Block Copolymers II, ed. A. H. E. Müller and O. Borisov, Springer, Heidelberg, 1st edn, 2011, vol. 242, ch. 1, pp. 1-28. 20 R. Rodriguez-Garcia, M. Mell, I. López-Montero, J. Netzel, T. Hellweg and F. Monroy, Soft Matter, 2011, 7, 1532-1542.

21 A. Caillé, C. R. Acad. Sci. Ser. B, 1972, 274, 891-893.

22 D. Roux and C. R. Safinya, J. Phys., 1988, 49, 307-318.

23 F. Nallet, R. Laversanne and D. Roux, J. Phys. II, 1993, 3, 487502.

24 D. Roux, C. R. Safinya and F. Nallet, in Micelles, Membranes, Microemulsions, and Monolayers, ed. W. M. Gelbart, A. BenShaoul and D. Roux, Springer Verlag, New York, 1994.

25 H. Egger, T. Hellweg and G. H. Findenegg, Phys. Chem. Chem. Phys., 2003, 5, 3013-3020.

26 A. G. Zilman and R. Granek, Phys. Rev. Lett., 1996, 77, 47884791.

27 O. Holderer, H. Frielinghaus, M. Monkenbusch, M. Klostermann, T. Sottmann and D. Richter, Soft Matter, 2013, 9, 2308-2313.

28 L. Rodriguez-Arriaga, I. Lopez-Montero, F. Monroy, G. O. Gil, B. Farago and T. Hellweg, Biophys. J., 2009, 96, 3629-3637.

29 L. Rodriguez-Arriaga, I. Lopez-Montero, G. O. Gil, B. Farago, T. Hellweg and F. Monroy, Phys. Rev. E: Stat., Nonlinear, Soft Matter Phys., 2009, 80, 031908.

30 M. Nonomura and T. Ohta, J. Chem. Phys., 1999, 110, 75167523.

31 M. Mihailescu, M. Monkenbusch, J. Allgaier, H. Frielinghaus, D. Richter, B. Jakobs and T. Sottmann, Phys. Rev. E: Stat., Nonlinear, Soft Matter Phys., 2002, 66, 1-13. 32 P. G. de Gennes, Physica, 1959, 25, 825. 